\title{
Sleep disorder in patients with chronic liver disease: a narrative review
}

\author{
Neeraj Mukesh Shah ${ }^{1,2,3}$, Akanksha Mimi Malhotra ${ }^{1}$, Georgios Kaltsakas ${ }^{1,2,3}$ \\ ${ }^{1}$ Lane Fox Respiratory Service, St Thomas' Hospital, Guy's and St Thomas' NHS Foundation Trust, London, UK; ${ }^{2}$ Lane Fox Clinical Respiratory \\ Physiology Centre, Guy's and St Thomas' NHS Foundation Trust, London, UK; ${ }^{3}$ Centre for Human and Applied Physiological Sciences (CHAPS), \\ King's College London, London, UK \\ Contributions: (I) Conception and design: NM Shah, G Kaltsakas; (II) Administrative support: All authors; (III) Provision of study materials or \\ patients: All authors; (IV) Collection and assembly of data: NM Shah, AM Malhotra; (V) Data analysis and interpretation: All authors; (VI) \\ Manuscript writing: All authors; (VII) Final approval of manuscript: All authors. \\ Correspondence to: Dr. Georgios Kaltsakas. Lane Fox Respiratory Unit, St Thomas Hospital, Westminster Bridge Road, London, SE1 7EH, UK. \\ Email: Georgios.kaltsakas@gstt.nhs.uk.
}

\begin{abstract}
Sleep disturbance is a common feature of chronic liver disease (CLD) with impact on healthrelated quality of life; $60-80 \%$ of patients with CLD report subjective poor sleep; frequent presentations of sleep disturbance include insomnia, reduced sleep efficiency, increased sleep latency, reduced time in rapid eye movement (REM) sleep, restless leg syndrome and excessive daytime sleepiness (EDS). Key contributors to sleep disturbance include hepatic encephalopathy $(\mathrm{HE})$ and circadian rhythm imbalance due to altered melatonin metabolism. Specific conditions causing CLD, such as non-alcoholic fatty liver disease (NAFLD), chronic viral hepatitis and primary biliary cholangitis (PBC) result in different types of sleep disturbance, and the treatment of these conditions can often also lead to sleep disturbance. There are currently limited management options for sleep disturbance in CLD. Obstructive sleep apnoea (OSA) is a common condition that causes chronic intermittent hypoxia due to airway collapse during sleep. This chronic intermittent hypoxia appears to contribute to the development of NAFLD. The presence of reactive oxygen species and the overexpression of hypoxia inducible factor 1-alpha secondary to hypoxia may be responsible for the second 'hit' of the 'two-hit' hypothesis of NAFLD. Treatment of the intermittent hypoxia with continuous positive airway pressure therapy has limited efficacy against liver dysfunction. There remain many outstanding areas of investigation in the management of sleep disturbance in CLD, and of liver dysfunction in OSA.
\end{abstract}

Keywords: Chronic liver disease; cirrhosis; non-alcoholic fatty liver disease; sleep disturbance; obstructive sleep apnoea

Submitted Jul 11, 2020. Accepted for publication Sep 10, 2020.

doi: $10.21037 /$ jtd-cus-2020-012

View this article at: http://dx.doi.org/10.21037/jtd-cus-2020-012

\section{Introduction}

The association between sleep disturbance and chronic liver disease (CLD) is increasingly recognized as an important part of the disease course of patients with CLD. Sleep disturbance in patients with cirrhosis is independently associated with reduced health-related quality of life $(1,2)$. In addition, patients with sleep disorders may be at increased risk of developing liver malignancy (3); it is therefore an important area to review. This review will discuss the prevalence and character of sleep disturbance in CLD and then describe possible pathophysiological mechanisms causing sleep disturbance within this population. The limited management options currently available for sleep disturbance in CLD will then be described. Finally, liver dysfunction in obstructive sleep apnoea (OSA), and particularly the association with non-alcoholic fatty liver disease (NAFLD), will be explored. This review will focus 
on the respiratory contribution to the development of sleep disturbance in CLD. While it is recognised that neurology has an important contribution, it is beyond the scope of this review to discuss this. Many clinical and research questions remain unanswered in this field and this review highlights potential future avenues for investigation.

We present the following article in accordance with the Narrative Review Checklist (available at http://dx.doi. org/10.21037/jtd-cus-2020-012).

\section{Methods}

Literature for this narrative review was identified using the following terms in Medline and Embase: liver, chronic, sleep, excessive daytime sleepiness, non-alcoholic fatty liver disease, cirrhosis, obstructive sleep apnoea, respiratory and pulmonary. There were no restrictions on dates or study design. English language articles were included.

\section{Sleep disturbance in CLD}

Sleep disturbance is relatively common in patients with CLD and cirrhosis; $60-80 \%$ of patients with cirrhosis rated themselves as poor sleepers, according to the Pittsburgh Sleep Quality Index (PSQI). PSQI was higher in those patients with evidence of hepatic encephalopathy (HE) and those with increased PSQI suffered from worse health-related quality of life $(1,4)$. Almost $50 \%$ of patients with cirrhosis without HE reported unsatisfactory sleep. Nocturnal actigraphy and polysomnography studies have demonstrated short sleeping time ( $<6$ hours per night), reduced sleep efficiency, increased sleep latency, increased rapid eye movement (REM) latency and reduced REM sleep, frequent nocturnal awakening, and excessive daytime sleepiness (EDS) $(5,6)$. Excessive periodic limb movements during sleep have also been reported (7); $25-40 \%$ of cirrhotic patients reported insomnia, with increased prevalence of insomnia with worsening severity of cirrhosis. In addition, patients reporting insomnia also reported higher EDS $(8,9)$. It is therefore important to screen for sleep disturbance in any patient known to have or presenting with CLD, including cirrhosis. There are currently no specific tools to assess sleep quality in CLD. The PSQI remains the gold-standard self-reported tool and the Epworth Sleepiness Scale can be used to assess EDS. Objective assessment of sleep quality involves polysomnography or nocturnal actigraphy, and their feasibility in liver disease patients has been demonstrated (10).

\section{Pathophysiology of sleep disturbance in CLD}

The cause of sleep disturbance in CLD is likely to be multifactorial and has not been fully elicited. Several mechanisms have been observed that can be associated with poor sleep and are proposed here as possible causes of sleep disturbance.

A common feature of sleep disturbance in CLD is delayed sleep onset. Disruption of the melatonin metabolism pathway is a potential cause for this. The liver plays a central role in the metabolism of melatonin, as it is predominantly metabolized by cytochrome $\mathrm{P}_{450}$ (11). Normal melatonin rhythm is disturbed in patients with CLD, likely due to impaired melatonin clearance in liver dysfunction $(12,13)$. The cause of this impaired melatonin clearance is unclear but may be related to decreased hepatic blood flow and competition with bilirubin in the intrahepatic transport system (14). Patients with cirrhosis have elevated daytime melatonin levels and a delayed onset of melatonin peak at night $(12,13,15)$. Elevated daytime melatonin causes a biological clock phase-shift and this disruption to the circadian rhythm may be related to sleep disturbance in CLD $(13,16)$. Indeed, disrupted melatonin rhythm has been associated with delayed sleep onset in cirrhosis (17). Importantly, these melatonin abnormalities have been observed in patients without significant $\mathrm{HE}$, demonstrating that this derangement is independent of HE (14). A case report has demonstrated that melatonin rhythm can be corrected back to normal values following a liver transplant, providing further evidence for the role of liver dysfunction in melatonin disruption (18). Another possible cause of delayed sleep onset is disturbed regulation of core body temperature. Sleep onset relies on core body temperature cooling through thermoregulatory mechanisms (19). Distal/proximal skin temperature and their gradient (DPG) are reliable predictors of sleep latency; the greater the peripheral vasodilatation, the shorter the time taken to fall asleep (20). A cohort of cirrhotic patients demonstrated higher proximal temperature and significantly lower DPG than healthy controls, and these changes were correlated with an altered sleep-wake profile (21). A potential cause for this impaired cooling is splanchnic and systemic vasodilatation, due to a hyperdynamic circulation (22), causing peripheral vasoconstriction and cooler peripheries.

Another common feature of sleep disturbance in CLD is EDS. EDS is widely recognized as a feature of HE $(2,5,8,23,24)$. EDS, assessed by the Epworth Sleepiness Scale, is well correlated with the 
degree of HE $(2,4)$ and the absence of EDS is useful in predicting an HE-free follow-up period (24). Furthermore, an ammonia load, simulating HE, resulted in increased subjective EDS in cirrhosis patients compared to healthy controls (25). Of note, although HE was associated with EDS, it has not been associated with night-time sleep disturbance (2). However, the study cohort consisted primarily of patients with alcoholic-related- and hepatitis C-related cirrhosis. With the advent of NAFLD and its associated metabolic conditions such as type II diabetes, hypertension and OSA, the night-time sleep profile may be different. Critical flicker frequency is the frequency at which a flickering light is indistinguishable from a steady state light; it has been demonstrated to be a reliable measure to identify minimal HE (26). A case-control study demonstrated that cirrhotic patients with higher evening critical flicker frequency (and therefore evidence of minimal HE) suffered from sleep disturbance, more commonly than cirrhotic patients with lower evening critical flicker frequency and controls (27). It therefore seems that there is a need to better understand the diurnal variation in $\mathrm{HE}$ and its potential contribution to sleep disturbance.

The cause of disturbed sleep in patients with CLD remains unclear. Various cellular mechanisms have been proposed with limited evidence to support them. In a cohort of alcohol-unrelated cirrhosis patients, raised serum interleukin-6 (IL-6) was identified as an independent predictor of disturbed sleep, measured by PSQI (28). IL-6 is associated with sleep conditions such as OSA and narcolepsy $(29,30)$. Although the effect of disturbed sleep has been demonstrated on IL-6 $(31,32)$, the direct effect of IL-6 levels on sleep quality has not been clearly elicited. The existing evidence does suggest, however, that there is an association between sleep quality and IL-6. Another independent risk factor for disturbed sleep is blood glucose fluctuation. Abnormal glucose fluctuations were associated with higher PSQI scores in a cohort of patients with CLD (33). As $60-70 \%$ of patients with CLD can have impaired glucose intolerance $(34,35)$, and sleep disturbance is associated with glucose intolerance (36), it would be unsurprising if fluctuating glucose levels contribute to poor sleep in CLD. Recently, sarcopenia and associated decreased skeletal muscle strength have been demonstrated to be independent risk factors for disturbed sleep quality (measured by PSQI), in a cohort of patients with CLD (37). Unlike many other instances of sleep disturbance, hypoventilation does not appear to be a contributory factor. Patients with cirrhosis do not hypoventilate (38), and may in fact hyperventilate (39).
The presence of ascites may be expected to cause a degree of hypoventilation due to mechanical compression of the diaphragm. Indeed, following abdominal paracentesis, lung volumes increase (40); however respiratory muscle strength is preserved even with ascites (41) and so it is unlikely that clinically significant hypoventilation is present.

The pathophysiology of sleep disturbance in CLD remains unclear with several different mechanisms proposed. It is particularly difficult to elicit the exact mechanism as the mechanisms proposed can often contribute to sleep disturbance in all patients, not only those with CLD, and the literature does not delineate this well. It is likely that sleep disturbance in CLD is a multifactorial phenomenon and may be disease-specific (Table 1) and so requires tailored means to manage it.

\section{NAFLD}

NAFLD is a common condition, prevalent in $25 \%$ of the global population and increasing as the prevalence of obesity increases (42). NAFLD is characterized by fatty deposition in the liver in the absence of any secondary causes of fatty deposition (43). Increased fat deposition in the liver causes inflammation and fibrosis, eventually leading to cirrhosis. Sleep disruption is increased in patients with NAFLD compared to healthy controls, with NAFLD patients reporting higher PSQI than control (44). Although a meta-analysis reported no relationship between sleep duration and NAFLD (45), subsequent cohort studies have continued to suggest that short sleep duration increases the risk of NAFLD (46-48). Conversely, optimal sleep duration (7-9 hours) has been negatively correlated with liver stiffness in NAFLD patients (49). The most common forms of sleep disturbance in NAFLD include OSA, insomnia and restless leg syndrome $(50,51)$.

\section{Chronic viral hepatitis}

Sleep disturbance affects up to $60 \%$ of untreated patients with chronic hepatitis C (HCV) $(52,53)$ and is one of the most commonly reported symptoms (54). When compared to healthy controls, HCV patients reported significantly worse sleep quality, assessed by PSQI and actigraphy (55). $\mathrm{HCV}$ patients suffered from more awakenings, and worse sleep efficiency and latency. Unlike other causes of cirrhosis, sleep disturbance in HCV is independent of the extent of fibrosis (56). Treatment with direct antiviral agents appears to improve subjective sleep quality (57), while 
Table 1 Specific forms of chronic liver disease and the features of sleep disturbance that patients most frequently suffer from. Potential specific treatments for sleep disturbance under investigation are also provided, although the evidence base supporting these is of mixed quality

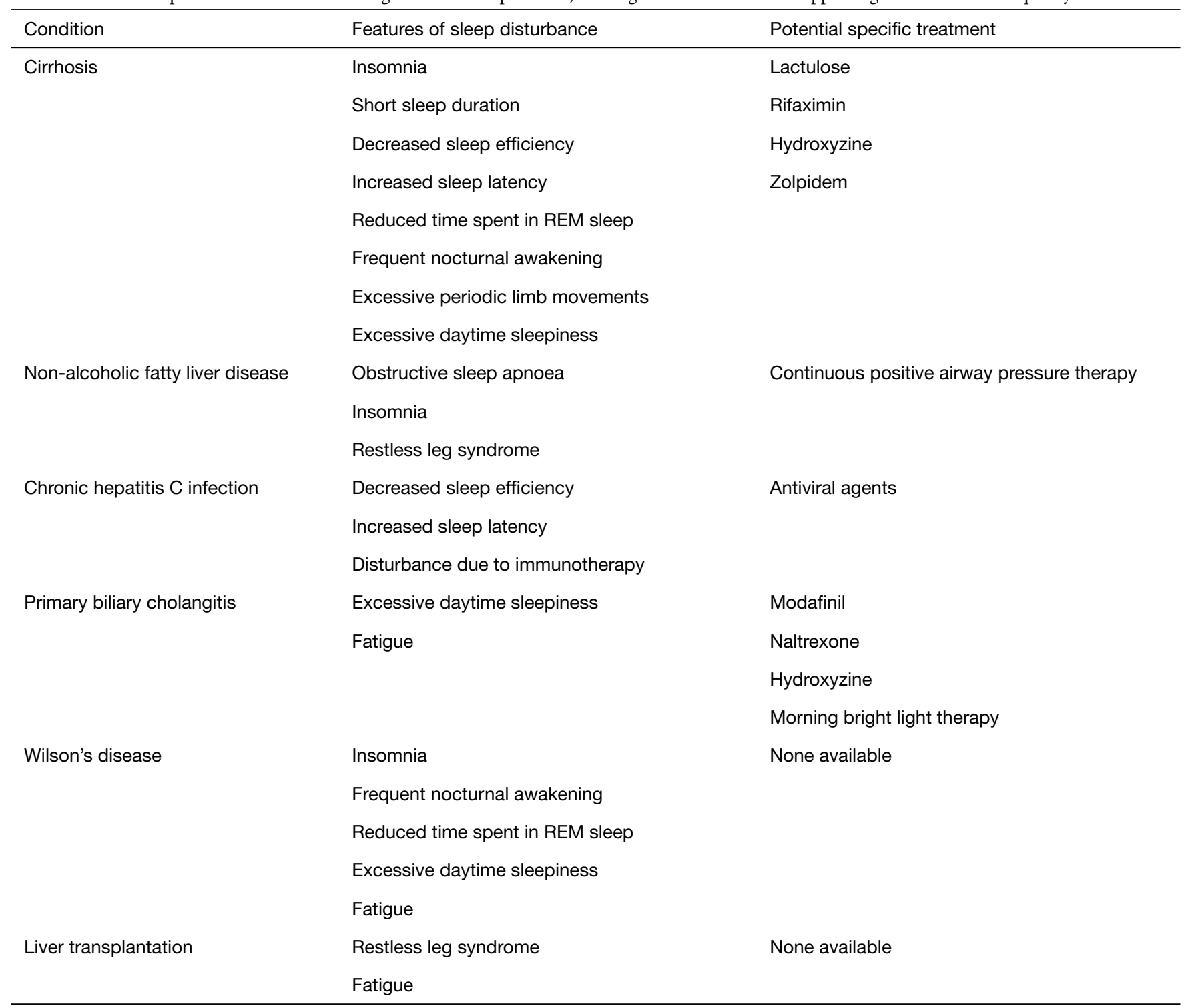

immunotherapy with interferon therapy appears to increase the risk of sleep disturbance. Patients receiving interferon therapy suffered from worse subjective sleep quality when compared to those not receiving any treatment (58). They also suffered from worse actigraphy markers of sleep quality than patients receiving direct antiviral treatment without interferon therapy (59). The addition of resveratrol (a natural phenol) to interferon therapy appears to improve subjective sleep quality (60).

The impact of chronic hepatitis B infection (HBV) on sleep quality is less clear. A cohort study has reported that $60 \%$ of $\mathrm{HBV}$ patients reported poor sleep quality (PSQI >5) (61), while a case-control study reported that $64 \%$ of HBV patients reported symptoms of insomnia compared to $35 \%$ in healthy controls (62). No literature has been identified reporting the impact of interventions on sleep quality in HBV.

\section{Primary biliary cholangitis (PBC)}

$\mathrm{PBC}$, formerly known as primary biliary cirrhosis, is an autoimmune condition resulting in progressive destruction 
of the biliary system, ultimately leading to cirrhosis. Prevalence of PBC is 35 per 100,000 population (63). Patients are likely to suffer from fatigue, pruritus and EDS; these symptoms have a profound effect of quality of life (64). When compared to healthy controls, PBC patients suffer from greater EDS (measured by Epworth Sleepiness Scale) and worse sleep quality (measured as PSQI) (65). Self-reported sleep timing and quality are worse in PBC compared to healthy controls and pruritus appears to coincide with feeling of sleepiness (66). Modafinil has been investigated as a treatment for EDS in PBC. Although an open-label study demonstrated improvement in ESS following modafinil administration $(67,68)$, a subsequent randomized trial investigating the effect of modafinil on fatigue demonstrated no benefit (69). There is therefore no clear consensus on the value of modafinil in PBC. An alternative approach that has been investigated to improve sleep is to target pruritus. Naltrexone, an opiate receptor antagonist used to treat pruritus, appears to improve subjective sleep quality (70). Hydroxyzine, an antihistamine, results in significant improvement in subjective and objective sleep quality, although whether this directly acts on pruritus, or provides benefit through sedation is not clear (71). Morning bright light therapy appears to have beneficial effect on subjective sleep quality as well as actigraphy markers of sleep quality, as well as quality of life (72). Despite these potential treatments, a recent meta-analysis investigating treatments for fatigue in $\mathrm{PBC}$ were unable to identify any beneficial intervention aside from liver transplantation (73).

\section{Wilson's disease (WD)}

WD is an autosomal recessive disorder of copper metabolism, characterized by excessive accumulation of copper in the liver, brain (basal ganglia) and cornea. This manifests as hepatic failure, and neuropsychiatric and parkinsonian symptoms. Sleep disturbance in WD has been well described. The most common symptoms of sleep disturbance include EDS, frequent nocturnal awakening, difficulty falling asleep and fatigue (74-77). Polysomnographic assessment reveals that these patients suffer from reduced total sleep time, sleep efficiency, time spent in REM sleep, and prolonged sleep onset latency (74). Subjective assessments of sleep quality have also demonstrated poorer sleep quality when compared to age-matched controls (77), and these correlate well with polysomnographic studies (76). Chelating therapy with
$\mathrm{D}$-penicillamine is the mainstay of treatment for WD. Patients receiving D-penicillamine suffered from prolonged REM sleep-onset latency (74) and reported a higher incidence of REM behaviour disorders, such as vivid dreams and cataplexy-like episodes than age-matched controls (76).

\section{Liver transplantation}

As liver transplantation reverses many of the effects of CLD, it may be assumed that following transplantation, there will be an improvement in sleep quality. Prior to transplantation, $60-70 \%$ of candidates for transplantation reported poor sleep quality $(78,79)$. Following transplantation, 50-80\% of transplant recipients reported poor sleep quality (79-81). One study reported that poor sleep quality was reported by only $40 \%$ of patients (82), however this study reported poor sleep quality as PSQI $>7$, whereas other studies report poor sleep quality as PSQI $>5$, so they are likely to have underreported their proportion patients reporting poor sleep. It therefore appears that liver transplantation does not have a positive impact on the prevalence of sleep disturbance. Sleep disturbance after liver transplantation has been correlated with anxiety and depression $(82,83)$, the presence of minimal HE and restless leg syndrome (81) and increased fatigue levels (79). Importantly, sleep disturbance was significantly correlated with reduce health-related quality of life in these patients (81). The underlying cause of CLD may also have relevance to the presence of sleep disturbance; patients with alcohol-related liver disease were significantly more likely to report an improvement in sleep disturbance following transplantation, whereas patients with HCV did not report any difference in sleep disturbance (84).

\section{Management of sleep disturbance in CLD}

Limited interventions have been investigated specifically targeting sleep disturbance in CLD. Lactulose is widely used to treat HE. A randomized study demonstrated a significant improvement in subjective sleep quality after three months of lactulose in cirrhotic patients with minimal HE (85). An observational study investigating threemonth lactulose therapy in minimal HE patients revealed improvements in PSQI, Epworth Sleepiness Scale and polysomnographic measures of sleep quality (total sleep time, sleep efficiency, sleep latency and amount of REM sleep) (86). Rifaximin, an antibiotic used for refractory HE has been demonstrated to improve the amount of time spent in REM sleep, without changes to EDS or subjective 


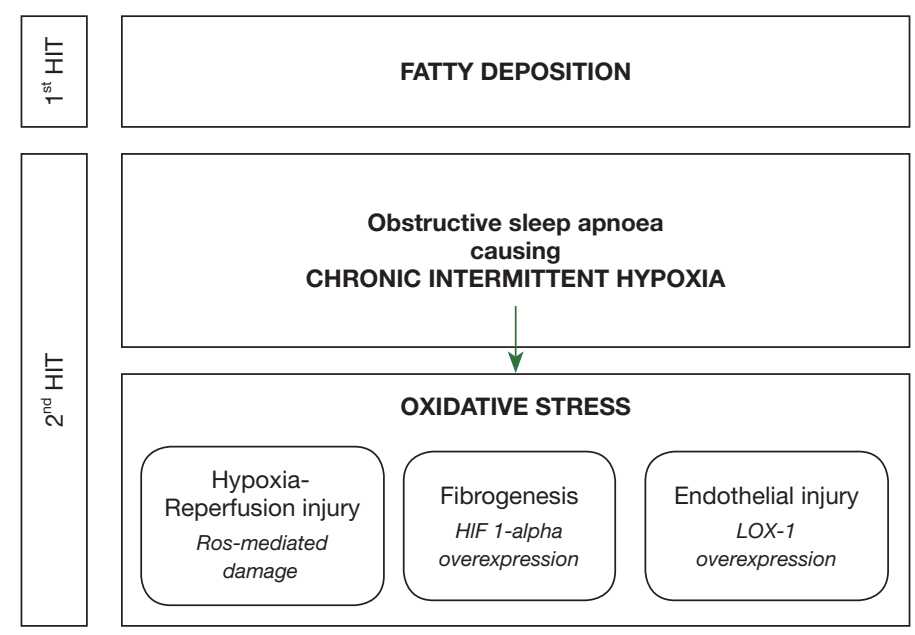

Figure 1 The contribution of obstructive sleep apnoea to the pathophysiology of non-alcoholic fatty liver disease (NAFLD). Following the first 'hit' of fatty liver deposition, the chronic intermittent hypoxia of obstructive sleep apnoea contributes to endothelial damage that contributes to the fibrotic change seen in NAFLD. ROS, reactive oxygen species; HIF 1-alpha, hypoxia inducible factor 1-alpha; LOX-1, lectin-like oxidized low-density lipoprotein receptor-1.

sleep quality (87). A randomized trial investigating the effect of short-term hydroxyzine in patients with cirrhosis demonstrated an improvement in sleep efficiency and a subjective improvement in sleep quality (71). A randomized study investigating a month's treatment of zolpidem in patients with cirrhosis revealed improvements in total sleep time, sleep efficiency, sleep latency and the number of arousals and subjective sleep quality (PSQI), without any changes to sleep architecture (88). Interestingly, given the close association between melatonin rhythm and sleep disturbance in CLD, the administration of melatonin has not been specifically investigated. Light therapy has been investigated as a means to correct circadian rhythm, but despite positive case report evidence (89), a randomized trial failed to demonstrate any benefit (90).

\section{OSA and NAFLD}

Having reviewed the presence of sleep disturbance in CLD, we now consider the presence of liver dysfunction in OSA. OSA is a relatively common respiratory disorder, with an estimated global prevalence of clinically relevant OSA of $6 \%$ (91). It is closely related to obesity and has close association with other metabolic conditions such as type II diabetes mellitus. It is therefore unsurprising that it is also closely associated with NAFLD (92). Secondary to this, liver dysfunction is often present in patients with OSA (93). Studies investigating the prevalence of liver dysfunction in
OSA have reported that OSA is independently associated with elevated transaminases (94-96), and progression to non-alcoholic steatohepatitis (NASH) and liver fibrosis (94,97-99). In addition, in obese patients, the absence of OSA was significantly associated with normal liver histology (100), further demonstrating the close association between OSA and NAFLD. There are limited data reporting liver function in obesity hypoventilation syndrome; a single study has reported that when compared with OSA, patients with obesity hypoventilation syndrome have a significantly higher serum alkaline phosphatase level (101). However, when compared with OSA patients with a body mass index $>30$, there was no difference, and so this effect on liver function may have been related to obesity rather than sleep-disordered breathing per se.

The pathophysiology of NAFLD, and its progression to NASH and fibrosis, is widely explained by the 'twohit hypothesis'. The first 'hit' is the build-up of lipids in hepatocytes. The second 'hit' then causes hepatocyte injury, inflammation and eventual fibrosis, and can be initiated by oxidative stress, proinflammatory cytokines or mitochondrial dysfunction (102). Clear understanding of the second 'hit' and its antecedents remains to be discovered. The literature is suggestive that the chronic intermittent hypoxia of OSA is an important contributor to this second 'hit' (Figure 1). In patients with OSA, the overnight desaturation index and mean nocturnal oxygen saturation are independent risk factors for the development of liver injury, indicated 
by rising transaminases $(103,104)$, NAFLD (105), and hepatic fibrosis $(106,107)$. Similarly, patients with NAFLD have a higher apnoea-hypopnoea index and lower mean nocturnal oxygen saturation than controls $(108,109)$, and these are independent risk factors for progression to hepatic fibrosis $(110,111)$. In addition, increasing severity of OSA appears to be in a dose-relationship with the prevalence of NAFLD (112) and severity of liver dysfunction, both in terms of transaminases $(113,114)$ and liver morphology $(115-117)$.

Human studies have not clearly demonstrated the pathophysiological impact of chronic intermittent hypoxia on liver function, so our current understanding comes from animal models. In a mouse model, intermittent hypoxia resulted in a cyclic reduction in oxygen tension in the liver (118), suggesting that it causes cycles of hypoxia and reoxygenation, which may result in oxidative stress from reactive oxygen species, similar to hypoxia-reperfusion injury. Contiguous to this, many human studies have demonstrated that OSA can cause high levels of oxidative stress $(119,120)$. Despite this, two studies investigating the utility of the antioxidants, vitamin C (121) and carbocisteine (122), were not able to provide strong evidence for their use in improving OSA outcomes. Hypoxiainducible factor 1-alpha (HIF 1-alpha) is a transcription factor that regulates the cellular response to hypoxia. In a mouse model of NAFLD, deletion of the HIF 1-alpha gene protected hepatocytes from progression to liver fibrosis $(123,124)$. Pathological angiogenesis in the liver is closely associated with the fibrogenic progression of CLDs to fibrosis. Both hypoxia and HIF 1-alpha act as proangiogenic stimuli so appear to be a key contributor to the fibrotic changes observed with progressive NAFLD (125). Lectin-like oxidized low-density lipoprotein receptor-1 $(\mathrm{LOX}-1)$ is a cell surface protein that facilitates the endocytosis of oxidized lipoproteins in endothelial cells. The deletion of HIF 1-alpha downregulates the expression of LOX-1 (126) and so it can be surmised that overexpression of HIF 1-alpha, such as in OSA will result in overexpression of LOX-1. Indeed, a cohort of treatmentnaïve OSA patients demonstrated that LOX-1 levels were higher in patients with OSA than control and that the proportion of sleep spent with oxygen saturations below $90 \%$ was positively correlated with LOX-1 levels (127). Importantly, in vitro data have revealed that LOX-1 expression is associated with endothelial injury and the defenestration of human liver sinusoidal endothelial cells, which is a pathological feature of NAFLD (128). Based on this mechanistic data, the progression of NAFLD to NASH and cirrhosis appears to be mediated by HIF 1 -alpha and its effect on LOX-1 expression, which is released in response to the chronic intermittent hypoxia caused by OSA.

We could therefore posit that treating the intermittent hypoxia of OSA, by continuous positive airway therapy (CPAP), would be protective against liver dysfunction in OSA patients. A meta-analysis that identified limited data concluded that CPAP is effective at improving serum liver enzyme levels in patients with NAFLD (129). Subsequently published data support this finding $(130,131)$. In addition, CPAP therapy appears to delay progression from NAFLD to liver fibrosis (132). Despite this, and although hepatic steatosis is a reversible condition, CPAP does not appear to improve morphological changes in the liver in OSA patients $(133,134)$. A meta-analysis of five randomised studies has also concluded that CPAP does not appear to have any effect on liver fibrosis and that the existing evidence for this is of low quality (135).

The relationship between NAFLD and OSA is clearly complex and causality remains uncertain; the hypoxia of OSA does appear to contribute to the development of NAFLD and to the progression of NAFLD to liver fibrosis. However, eliminating this hypoxia does not result in a complete reversal of the morphological liver changes and so there are likely to be contributions from other mechanisms that remain to be discovered. There are also outstanding clinical questions that need to be answered through further work. The clinical benefit of screening all NAFLD patients for OSA and vice versa remains unclear, but we would recommend the inclusion of simple screening tools such as questionnaires (OSA) and liver function tests (NAFLD) in the baseline work-up of these patients, as suggested by the authors of a meta-analysis investigating abnormal liver enzymes in OSA (136). There is increasing interest in biomarkers of liver fibrosis in OSA $(137,138)$; if a simple non-invasive biomarker can be identified, this would further support the rationale to screen all OSA patients for liver dysfunction. If evidence of liver dysfunction is present, should an OSA patient be treated more aggressively with earlier initiation of CPAP than in patients without liver dysfunction? Clinical trials are needed to address the correct timing of CPAP initiation in the context of liver dysfunction. Other treatment of OSA, such as the mandibular device, also aim to eliminate the intermittent hypoxia. The role that treatments for OSA, other than CPAP, have in improving liver dysfunction is yet to be investigated. 


\section{Summary}

Sleep disturbance in CLD is common and associated with reduced health-related quality of life. It can manifest in various forms, including delayed sleep onset, EDS and poor subjective sleep quality. The causes are complex, multifactorial and remain to be elucidated clearly. Specific conditions leading to CLD manifest sleep disturbance in different ways, and surprisingly, liver transplantation does not result in an improvement in sleep quality. There are currently minimal management options specifically for sleep disturbance in patients with CLD, with the most convincing evidence available for established treatments of $\mathrm{HE}$, such as lactulose and rifaximin. There is a need to conduct more randomised trials to identify useful pharmacological agents specifically aimed at improving sleep quality. NAFLD is closely associated with OSA; mechanistic studies and animal models provide a potential pathophysiological explanation for the contribution of OSA to the progression of NAFLD to liver fibrosis and cirrhosis, through chronic intermittent hypoxia. Despite this, treatment of intermittent hypoxia with CPAP does not result in a significant reversal of progressive liver disease in NAFLD. There is therefore a need to further identify the exact contribution of OSA to progressive NAFLD, the optimal tool and time point to screen for OSA in NAFLD and for NAFLD in OSA, and whether the timing of CPAP initiation has any impact on the progression of NAFLD.

\section{Acknowledgments}

Funding: None.

\section{Footnote}

Provenance and Peer Review: This article was commissioned by the Guest Editor (Joerg Steier) for the series " $5^{\text {th }}$ Clinical Update Sleep" published in Fournal of Thoracic Disease. The article was sent for external peer review.

Reporting Checklist: The authors have completed the STROBE reporting checklist. Available at http://dx.doi. org/10.21037/jtd-cus-2020-012

Conflicts of Interest: All authors have completed the ICMJE uniform disclosure form (available at http://dx.doi. org/10.21037/jtd-cus-2020-012). The series " $5^{\text {th }}$ Clinical Update Sleep” was commissioned by the editorial office without any funding or sponsorship. The authors have no other conflicts of interest to declare.

Ethical Statement: The authors are accountable for all aspects of the work in ensuring that questions related to the accuracy or integrity of any part of the work are appropriately investigated and resolved.

Open Access Statement: This is an Open Access article distributed in accordance with the Creative Commons Attribution-NonCommercial-NoDerivs 4.0 International License (CC BY-NC-ND 4.0), which permits the noncommercial replication and distribution of the article with the strict proviso that no changes or edits are made and the original work is properly cited (including links to both the formal publication through the relevant DOI and the license). See: https://creativecommons.org/licenses/by-nc-nd/4.0/.

\section{References}

1. Ghabril M, Jackson M, Gotur R, et al. Most Individuals With Advanced Cirrhosis Have Sleep Disturbances, Which Are Associated With Poor Quality of Life. Clin Gastroenterol Hepatol 2017;15:1271-8.e6.

2. Montagnese S, Middleton B, Skene DJ, et al. Night-time sleep disturbance does not correlate with neuropsychiatric impairment in patients with cirrhosis. Liver Int 2009;29:1372-82.

3. Liang JA, Sun LM, Muo CH, et al. Non-apnea sleep disorders will increase subsequent liver cancer risk--a nationwide population-based cohort study. Sleep Med 2012;13:869-74.

4. Samanta J, Dhiman RK, Khatri A, et al. Correlation between degree and quality of sleep disturbance and the level of neuropsychiatric impairment in patients with liver cirrhosis. Metab Brain Dis 2013;28:249-59.

5. Córdoba J, Cabrera J, Lataif L, et al. High prevalence of sleep disturbance in cirrhosis. Hepatology 1998;27:339-45.

6. Teodoro VV, Bragagnolo MA Jr, Lucchesi LM, et al. Polysomnographic sleep aspects in liver cirrhosis: a case control study. World J Gastroenterol 2013;19:3433-8.

7. Saleh K, Javaheri S. Sleep in ambulatory patients with stable cirrhosis of the liver. Sleep Med 2018;41:15-9.

8. Mostacci B, Ferlisi M, Baldi Antognini A, et al. Sleep disturbance and daytime sleepiness in patients with cirrhosis: a case control study. Neurol Sci 2008;29:237-40.

9. Al-Jahdali H, Al Enezi A, Anwar AE, et al. Prevalence of Insomnia and Sleep Patterns among Liver Cirrhosis 
Patients. J Circadian Rhythms 2014;12:2.

10. Formentin C, Garrido M, Montagnese S. Assessment and Management of Sleep Disturbance in Cirrhosis. Curr Hepatol Rep 2018;17:52-69.

11. Hardeland R. Melatonin metabolism in the central nervous system. Curr Neuropharmacol 2010;8:168-81.

12. Iguchi H, Kato KI, Ibayashi H. Melatonin serum levels and metabolic clearance rate in patients with liver cirrhosis. J Clin Endocrinol Metab 1982;54:1025-7.

13. Chojnacki C, Wachowska-Kelly P, Błasiak J, et al. Melatonin secretion and metabolism in patients with hepatic encephalopathy. J Gastroenterol Hepatol 2013;28:342-7.

14. Velissaris D, Karamouzos V, Polychronopoulos P, et al. Chronotypology and melatonin alterations in minimal hepatic encephalopathy. J Circadian Rhythms 2009;7:6.

15. Steindl PE, Finn B, Bendok B, et al. Disruption of the diurnal rhythm of plasma melatonin in cirrhosis. Ann Intern Med 1995;123:274-7.

16. Blei AT, Zee P. Abnormalities of circadian rhythmicity in liver disease. J Hepatol 1998;29:832-5.

17. Montagnese S, Middleton B, Mani AR, et al. Sleep and circadian abnormalities in patients with cirrhosis: features of delayed sleep phase syndrome? Metab Brain Dis 2009;24:427-39.

18. Cordoba J, Steindl P, Blei AT. Melatonin Arrhythmia Is Corrected After Liver Transplantation. Am J Gastroenterol 2009;104:1862-3.

19. Harding EC, Franks NP, Wisden W. The Temperature Dependence of Sleep. Front Neurosci 2019;13:336.

20. Kräuchi K, Cajochen C, Werth E, et al. Warm feet promote the rapid onset of sleep. Nature 1999;401:36-7.

21. Garrido M, Saccardo D, De Rui M, et al. Abnormalities in the 24-hour rhythm of skin temperature in cirrhosis: Sleep-wake and general clinical implications. Liver In 2017;37:1833-42.

22. Bolognesi M, Di Pascoli M, Verardo A, et al. Splanchnic vasodilation and hyperdynamic circulatory syndrome in cirrhosis. World J Gastroenterol 2014;20:2555-63.

23. Sherlock S, Summerskill WH, White LP, et al. Portalsystemic encephalopathy; neurological complications of liver disease. Lancet 1954;267:454-7.

24. De Rui M, Schiff S, Aprile D, et al. Excessive daytime sleepiness and hepatic encephalopathy: it is worth asking. Metab Brain Dis 2013;28:245-8.

25. Bersagliere A, Raduazzo ID, Nardi $M$, et al. Induced hyperammonemia may compromise the ability to generate restful sleep in patients with cirrhosis. Hepatology
2012;55:869-78.

26. Kircheis G, Wettstein M, Timmermann L, et al. Critical flicker frequency for quantification of low-grade hepatic encephalopathy. Hepatology 2002;35:357-66.

27. Gencdal G, Gunsar F, Meral CE, et al. Diurnal changes of critical flicker frequency in patients with liver cirrhosis and their relationship with sleep disturbances. Dig Liver Dis 2014;46:1111-5.

28. Tsai CF, Chu CJ, Wang YP, et al. Increased serum interleukin-6, not minimal hepatic encephalopathy, predicts poor sleep quality in nonalcoholic cirrhotic patients. Aliment Pharmacol Ther 2016;44:836-45.

29. Okun ML, Giese S, Lin L, et al. Exploring the cytokine and endocrine involvement in narcolepsy. Brain Behav Immun 2004;18:326-32.

30. Vgontzas AN, Papanicolaou DA, Bixler EO, et al. Elevation of plasma cytokines in disorders of excessive daytime sleepiness: role of sleep disturbance and obesity. J Clin Endocrinol Metab 1997;82:1313-6.

31. Redwine L, Hauger RL, Gillin JC, et al. Effects of sleep and sleep deprivation on interleukin-6, growth hormone, cortisol, and melatonin levels in humans. J Clin Endocrinol Metab 2000;85:3597-603.

32. Shearer WT, Reuben JM, Mullington JM, et al. Soluble TNF-alpha receptor 1 and IL-6 plasma levels in humans subjected to the sleep deprivation model of spaceflight. J Allergy Clin Immunol 2001;107:165-70.

33. Haraguchi M, Miyaaki H, Ichikawa T, et al. Glucose fluctuations reduce quality of sleep and of life in patients with liver cirrhosis. Hepatol Int 2017;11:125-31.

34. Nishida T. Diagnosis and Clinical Implications of Diabetes in Liver Cirrhosis: A Focus on the Oral Glucose Tolerance Test. J Endocr Soc 2017;1:886-96.

35. Chao HW, Chao SW, Lin H, et al. Homeostasis of Glucose and Lipid in Non-Alcoholic Fatty Liver Disease. Int J Mol Sci 2019;20:298.

36. Gottlieb DJ, Punjabi NM, Newman AB, et al. Association of Sleep Time With Diabetes Mellitus and Impaired Glucose Tolerance. Arch Intern Med 2005;165:863-7.

37. Nishikawa H, Enomoto H, Yoh K, et al. Effect of Sarcopenia on Sleep Disturbance in Patients with Chronic Liver Diseases. J Clin Med 2018;8:16.

38. Kao CH, Huang CK, Tsai SC, et al. Evaluation of lung ventilation and alveolar permeability in cirrhosis. J Nucl Med 1996;37:437-41.

39. Krowka MJ. Pathophysiology of arterial hypoxemia in advanced liver disease. Liver Transpl Surg 1996;2:308-12.

40. Chang SC, Chang HI, Chen FJ, et al. Effects of ascites and 
body position on gas exchange in patients with cirrhosis. Proc Natl Sci Counc Repub China B 1995;19:143-50.

41. Kaltsakas G, Antoniou E, Palamidas AF, et al. Dyspnea and respiratory muscle strength in end-stage liver disease. World J Hepatol 2013;5:56-63.

42. Younossi Z, Anstee QM, Marietti M, et al. Global burden of NAFLD and NASH: trends, predictions, risk factors and prevention. Nat Rev Gastroenterol Hepatol 2018;15:11-20.

43. Puri P, Sanyal AJ. Nonalcoholic fatty liver disease: Definitions, risk factors, and workup. Clin Liver Dis (Hoboken) 2012;1:99-103.

44. Marin-Alejandre BA, Abete I, Cantero I, et al. Association between Sleep Disturbances and Liver Status in Obese Subjects with Nonalcoholic Fatty Liver Disease: A Comparison with Healthy Controls. Nutrients 2019; 11:322.

45. Shen N, Wang P, Yan W. Sleep Duration and the Risk of Fatty Liver Disease: A Systematic Review and Metaanalysis. Sci Rep 2016;6:31956.

46. Okamura T, Hashimoto Y, Hamaguchi M, et al. Short sleep duration is a risk of incident nonalcoholic fatty liver disease: a population-based longitudinal study. J Gastrointestin Liver Dis 2019;28:73-81.

47. Kim D, Kim HJ, Kushida CA, et al. Short Sleep Duration Is Associated With Abnormal Serum Aminotransferase Activities and Nonalcoholic Fatty Liver Disease. Clin Gastroenterol Hepatol 2018;16:588-90.

48. Peng K, Lin L, Wang Z, et al. Short sleep duration and longer daytime napping are associated with nonalcoholic fatty liver disease in Chinese adults. J Diabetes 2017;9:827-36.

49. Katsagoni CN, Papatheodoridis GV, Papageorgiou MV, et al. A "healthy diet-optimal sleep" lifestyle pattern is inversely associated with liver stiffness and insulin resistance in patients with nonalcoholic fatty liver disease. Appl Physiol Nutr Metab 2017;42:250-6.

50. Mir HM, Stepanova M, Afendy H, et al. Association of Sleep Disorders with Nonalcoholic Fatty Liver Disease (NAFLD): A Population-based Study. J Clin Exp Hepatol 2013;3:181-5.

51. Wijarnpreecha K, Thongprayoon C, Panjawatanan P, et al. Insomnia and risk of nonalcoholic fatty liver disease: A systematic review and meta-analysis. J Postgrad Med 2017;63:226-31.

52. Lang CA, Conrad S, Garrett L, et al. Symptom prevalence and clustering of symptoms in people living with chronic hepatitis C infection. J Pain Symptom Manage
2006;31:335-44.

53. Sockalingam S, Abbey SE, Alosaimi F, et al. A review of sleep disturbance in hepatitis C. J Clin Gastroenterol 2010;44:38-45.

54. Evon DM, Stewart PW, Amador J, et al. A comprehensive assessment of patient reported symptom burden, medical comorbidities, and functional well being in patients initiating direct acting antiviral therapy for chronic hepatitis C: Results from a large US multi-center observational study. PLoS One 2018;13:e0196908.

55. Heeren M, Sojref F, Schuppner R, et al. Active at night, sleepy all day--sleep disturbances in patients with hepatitis C virus infection. J Hepatol 2014;60:732-40.

56. Monaco S, Mariotto S, Ferrari S, et al. Hepatitis C virus-associated neurocognitive and neuropsychiatric disorders: Advances in 2015. World J Gastroenterol 2015;21:11974-83.

57. Karimi-Sari H, Hosseini MA, Nikjoo N, et al. Patientreported outcomes of sleep, mood and quality of life after treatment of chronic hepatitis $\mathrm{C}$ infection using directacting antiviral agents. Clin Microbiol Infect 2020;26:1093. e5-1093.e8.

58. Martins EC, Silva MC, De Oliveira JA, et al. Sleep disturbances in hepatitis $\mathrm{C}$ patients undergoing treatment: minisleep questionnaire as a screening tool. Minerva Gastroenterol Dietol 2015;61:125-30.

59. Yoh K, Nishikawa H, Enomoto H, et al. Comparison of sleep disorders in chronic hepatitis $\mathrm{C}$ patients treated with interferon-based therapy and direct acting antivirals using actigraphy. Hepatol Res 2016;46:1358-66.

60. Pennisi M, Bertino G, Gagliano C, et al. Resveratrol in Hepatitis C Patients Treated with Pegylated-Interferon-2b and Ribavirin Reduces Sleep Disturbance. Nutrients 2017;9:897.

61. Xiao G, Ye Q, Han T, et al. Study of the sleep quality and psychological state of patients with hepatitis B liver cirrhosis. Hepatol Res 2018;48:E275-E282.

62. Guo HM, Liu M, Xiang YT, et al. Insomnia in Adults With Chronic Hepatitis B, Liver Failure, and Cirrhosis: A Case-Control Study. Perspect Psychiatr Care 2017;53:67-72.

63. Hirschfield GM, Dyson JK, Alexander GJM, et al. The British Society of Gastroenterology/UK-PBC primary biliary cholangitis treatment and management guidelines. Gut 2018;67:1568.

64. Mells GF, Pells G, Newton JL, et al. Impact of primary biliary cirrhosis on perceived quality of life: the UK-PBC national study. Hepatology 2013;58:273-83. 
65. Newton JL, Gibson GJ, Tomlinson M, et al. Fatigue in primary biliary cirrhosis is associated with excessive daytime somnolence. Hepatology 2006;44:91-8.

66. Montagnese S, Nsemi LM, Cazzagon N, et al. Sleep-Wake profiles in patients with primary biliary cirrhosis. Liver Int 2013;33:203-9.

67. Jones DE, Newton JL. An open study of modafinil for the treatment of daytime somnolence and fatigue in primary biliary cirrhosis. Aliment Pharmacol Ther 2007;25:471-6.

68. Hardy T, MacDonald C, Jones DE, et al. A followup study of modafinil for the treatment of daytime somnolence and fatigue in primary biliary cirrhosis. Liver Int 2010;30:1551-2.

69. Silveira MG, Gossard AA, Stahler AC, et al. A Randomized, Placebo-Controlled Clinical Trial of Efficacy and Safety: Modafinil in the Treatment of Fatigue in Patients With Primary Biliary Cirrhosis. Am J Ther 2017;24:e167-e176.

70. Wolfhagen FH, Sternieri E, Hop WC, et al. Oral naltrexone treatment for cholestatic pruritus: A doubleblind, placebo-controlled study. Gastroenterology 1997;113:1264-9.

71. Spahr L, Coeytaux A, Giostra E, et al. Histamine H1 blocker hydroxyzine improves sleep in patients with cirrhosis and minimal hepatic encephalopathy: a randomized controlled pilot trial. Am J Gastroenterol 2007;102:744-53.

72. Turco M, Cazzagon N, Franceschet I, et al. Morning Bright Light Treatment for Sleep-Wake Disturbances in Primary Biliary Cholangitis: A Pilot Study. Front Physiol 2018;9:1530.

73. Lee JY, Danford CJ, Trivedi HD, et al. Treatment of Fatigue in Primary Biliary Cholangitis: A Systematic Review and Meta-Analysis. Dig Dis Sci 2019;64:2338-50.

74. Netto AB, Sinha S, Taly AB, et al. Sleep in Wilson's disease: a polysomnography-based study. Neurol India 2010;58:933-8.

75. Netto AB, Sinha S, Taly AB, et al. Sleep in Wilson's disease: Questionnaire based study. Ann Indian Acad Neurol 2011;14:31-4.

76. Nevsimalova S, Buskova J, Bruha R, et al. Sleep disorders in Wilson's disease. Eur J Neurol 2011;18:184-90.

77. Portala K, Westermark K, Ekselius L, et al. Sleep in patients with treated Wilson's disease. A questionnaire study. Nord J Psychiatry 2002;56:291-7.

78. Marques DM, Teixeira HR, Lopes AR, et al. Sleep Quality Assessment and Daytime Sleepiness of Liver Transplantation Candidates. Transplant Proc
2016;48:2356-60.

79. Rodrigue JR, Nelson DR, Reed AI, et al. Fatigue and sleep quality before and after liver transplantation. Prog Transplant 2010;20:221-33.

80. van Ginneken BT, van den Berg-Emons RJ, van der Windt A, et al. Persistent fatigue in liver transplant recipients: a two-year follow-up study. Clin Transplant 2010;24:E10-6.

81. Akahoshi M, Ichikawa T, Taura N, et al. Sleep disturbances and quality of life in patients after living donor liver transplantation. Transplant Proc 2014;46:3515-22.

82. Zhu X, Ming Y, Liu J, et al. Sleep Quality and Psychosocial Factors in Liver Transplant Recipients at an Outpatient Follow-Up Clinic in China. Ann Transplant 2020;25:e920984.

83. Mendes KD, Lopes AR, Martins TA, et al. Relevance of anxiety and stress levels on sleep quality after liver transplantation. Transplant Proc 2014;46:1822-6.

84. Bhat M, Wyse JM, Moodie E, et al. Prevalence and predictors of sleep disturbance among liver diseases in long-term transplant survivors. Can J Gastroenterol Hepatol 2015;29:440-4.

85. Prasad S, Dhiman RK, Duseja A, et al. Lactulose improves cognitive functions and health-related quality of life in patients with cirrhosis who have minimal hepatic encephalopathy. Hepatology 2007;45:549-59.

86. Singh J, Sharma BC, Puri V, et al. Sleep disturbances in patients of liver cirrhosis with minimal hepatic encephalopathy before and after lactulose therapy. Metab Brain Dis 2017;32:595-605.

87. Bruyneel M, Sersté T, Libert W, et al. Improvement of sleep architecture parameters in cirrhotic patients with recurrent hepatic encephalopathy with the use of rifaximin. Eur J Gastroenterol Hepatol 2017;29:302-8.

88. Sharma MK, Kainth S, Kumar S, et al. Effects of zolpidem on sleep parameters in patients with cirrhosis and sleep disturbances: A randomized, placebo-controlled trial. Clin Mol Hepatol 2019;25:199-209.

89. De Rui M, Gaiani S, Middleton B, et al. Bright times for patients with cirrhosis and delayed sleep habits: a case report on the beneficial effect of light therapy. Am J Gastroenterol 2011;106:2048-9.

90. De Rui M, Middleton B, Sticca A, et al. Sleep and circadian rhythms in hospitalized patients with decompensated cirrhosis: effect of light therapy. Neurochem Res 2015;40:284-92.

91. Benjafield AV, Ayas NT, Eastwood PR, et al. Estimation of the global prevalence and burden of obstructive sleep apnoea: a literature-based analysis. Lancet Respir Med 
2019;7:687-98.

92. Umbro I, Fabiani V, Fabiani M, et al. Association between non-alcoholic fatty liver disease and obstructive sleep apnea. World J Gastroenterol 2020;26:2669-81.

93. Chou TC, Liang WM, Wang CB, et al. Obstructive sleep apnea is associated with liver disease: a population-based cohort study. Sleep Med 2015;16:955-60.

94. Tanne F, Gagnadoux F, Chazouilleres O, et al. Chronic liver injury during obstructive sleep apnea. Hepatology 2005;41:1290-6.

95. Jouet P, Sabate JM, Maillard D, et al. Relationship between obstructive sleep apnea and liver abnormalities in morbidly obese patients: a prospective study. Obes Surg 2007;17:478-85.

96. Kallwitz ER, Herdegen J, Madura J, et al. Liver enzymes and histology in obese patients with obstructive sleep apnea. J Clin Gastroenterol 2007;41:918-21.

97. Campos GM, Bambha K, Vittinghoff E, et al. A clinical scoring system for predicting nonalcoholic steatohepatitis in morbidly obese patients. Hepatology 2008;47:1916-23.

98. Ulitsky A, Ananthakrishnan AN, Komorowski R, et al. A noninvasive clinical scoring model predicts risk of nonalcoholic steatohepatitis in morbidly obese patients. Obes Surg 2010;20:685-91.

99. Pulixi EA, Tobaldini E, Battezzati PM, et al. Risk of obstructive sleep apnea with daytime sleepiness is associated with liver damage in non-morbidly obese patients with nonalcoholic fatty liver disease. PLoS One 2014;9:e96349.

100. Corey KE, Misdraji J, Zheng H, et al. The absence of obstructive sleep apnea may protect against non-alcoholic fatty liver in patients undergoing bariatric surgery. PLoS One 2013;8:e62504.

101.Akashiba T, Akahoshi T, Kawahara S, et al. Clinical characteristics of obesity-hypoventilation syndrome in Japan: a multi-center study. Intern Med 2006;45:1121-5.

102.Paschos P, Paletas K. Non alcoholic fatty liver disease and metabolic syndrome. Hippokratia 2009;13:9-19.

103.Lin QC, Chen LD, Chen GP, et al. Association between nocturnal hypoxia and liver injury in the setting of nonalcoholic fatty liver disease. Sleep Breath 2015;19:273-80.

104. Trzepizur W, Boursier J, Mansour Y, et al. Association Between Severity of Obstructive Sleep Apnea and Blood Markers of Liver Injury. Clin Gastroenterol Hepatol 2016;14:1657-61.

105.Aron-Wisnewsky J, Minville C, Tordjman J, et al.
Chronic intermittent hypoxia is a major trigger for nonalcoholic fatty liver disease in morbid obese. J Hepatol 2012;56:225-33.

106. Mesarwi OA, Shin MK, Drager LF, et al. Lysyl Oxidase as a Serum Biomarker of Liver Fibrosis in Patients with Severe Obesity and Obstructive Sleep Apnea. Sleep 2015;38:1583-91.

107.Agrawal S, Duseja A, Aggarwal A, et al. Obstructive sleep apnea is an important predictor of hepatic fibrosis in patients with nonalcoholic fatty liver disease in a tertiary care center. Hepatol Int 2015;9:283-91.

108. Cakmak E, Duksal F, Altinkaya E, et al. Association Between the Severity of Nocturnal Hypoxia in Obstructive Sleep Apnea and Non-Alcoholic Fatty Liver Damage. Hepat Mon 2015; 15:e32655.

109. Turkay C, Ozol D, Kasapoglu B, et al. Influence of obstructive sleep apnea on fatty liver disease: role of chronic intermittent hypoxia. Respir Care 2012;57:244-9.

110.Mishra P, Nugent C, Afendy A, et al. Apnoeic-hypopnoeic episodes during obstructive sleep apnoea are associated with histological nonalcoholic steatohepatitis. Liver Int 2008;28:1080-6.

111.Polotsky VY, Patil SP, Savransky V, et al. Obstructive sleep apnea, insulin resistance, and steatohepatitis in severe obesity. Am J Respir Crit Care Med 2009;179:228-34.

112. Ding H, Huang JF, Xie HS, et al. The association between glycometabolism and nonalcoholic fatty liver disease in patients with obstructive sleep apnea. Sleep Breath 2019;23:373-8.

113.Atan D, Köseo lu S, Özcan KM, et al. Evaluation of Liver Functions Based on Serum Aminotransferase Enzyme Levels in Patients with Obstructive Sleep Apnea Syndrome. Indian J Otolaryngol Head Neck Surg 2019;71:1679-82.

114. Chen LD, Zhang LJ, Lin XJ, et al. Association between continuous positive airway pressure and serum aminotransferases in patients with obstructive sleep apnea. Eur Arch Otorhinolaryngol 2018;275:587-94.

115. Trzepizur W, Boursier J, Le Vaillant $M$, et al. Increased liver stiffness in patients with severe sleep apnoea and metabolic comorbidities. Eur Respir J 2018;51:1800601.

116. Benotti P, Wood GC, Argyropoulos G, et al. The impact of obstructive sleep apnea on nonalcoholic fatty liver disease in patients with severe obesity. Obesity 2016;24:871-7.

117. Bernsmeier C, Weisskopf DM, Pflueger MO, et al. Sleep Disruption and Daytime Sleepiness Correlating with Disease Severity and Insulin Resistance in Non-Alcoholic Fatty Liver Disease: A Comparison with Healthy Controls. 
PLoS One 2015;10:e0143293.

118. Reinke C, Bevans-Fonti S, Drager LF, et al. Effects of different acute hypoxic regimens on tissue oxygen profiles and metabolic outcomes. J Appl Physiol (1985) 2011;111:881-90.

119. Eisele HJ, Markart P, Schulz R. Obstructive Sleep Apnea, Oxidative Stress, and Cardiovascular Disease: Evidence from Human Studies. Oxid Med Cell Longev 2015;2015:608438.

120. Yamauchi M, Nakano H, Maekawa J, et al. Oxidative stress in obstructive sleep apnea. Chest 2005;127:1674-9.

121. Grebe M, Eisele HJ, Weissmann N, et al. Antioxidant Vitamin C Improves Endothelial Function in Obstructive Sleep Apnea. Am J Respir Crit Care Med 2006;173:897-901.

122. Wu K, Su X, Li G, et al. Antioxidant Carbocysteine Treatment in Obstructive Sleep Apnea Syndrome: A Randomized Clinical Trial. PLoS One 2016;11:e0148519.

123. Mesarwi OA, Shin MK, Bevans-Fonti S, et al. Hepatocyte Hypoxia Inducible Factor-1 Mediates the Development of Liver Fibrosis in a Mouse Model of Nonalcoholic Fatty Liver Disease. PLoS One 2016;11:e0168572.

124.Moon JO, Welch TP, Gonzalez FJ, et al. Reduced liver fibrosis in hypoxia-inducible factor-1alphadeficient mice. Am J Physiol Gastrointest Liver Physiol 2009;296:G582-92.

125. Bocca C, Novo E, Miglietta A, et al. Angiogenesis and Fibrogenesis in Chronic Liver Diseases. Cell Mol Gastroenterol Hepatol 2015;1:477-88.

126. Crucet M, Wüst SJ, Spielmann P, et al. Hypoxia enhances lipid uptake in macrophages: role of the scavenger receptors Lox1, SRA, and CD36. Atherosclerosis 2013;229:110-7.

127.Akinnusi ME, Laporta R, El-Solh AA. Lectin-Like Oxidized Low-Density Lipoprotein Receptor-1 Modulates Endothelial Apoptosis in Obstructive Sleep Apnea. Chest 2011;140:1503-10.

128.Zhang Q, Liu J, Liu J, et al. oxLDL induces injury and defenestration of human liver sinusoidal endothelial cells via LOX1. J Mol Endocrinol 2014;53:281-93.

129. Chen LD, Lin L, Zhang LJ, et al. Effect of continuous positive airway pressure on liver enzymes in

Cite this article as: Shah NM, Malhotra AM, Kaltsakas G. Sleep disorder in patients with chronic liver disease: a narrative review. J Thorac Dis 2020;12(Suppl 2):S248-S260. doi: 10.21037/ jtd-cus-2020-012 obstructive sleep apnea: A meta-analysis. Clin Respir J 2018;12:373-81.

130. Kim D, Ahmed A, Kushida C. Continuous Positive Airway Pressure Therapy on Nonalcoholic Fatty Liver Disease in Patients With Obstructive Sleep Apnea. J Clin Sleep Med 2018;14:1315-22.

131. Khrisanapant W, Wuttiumporn K, Pasurivong O, et al. Liver function following continuous positive airway pressure therapy in obstructive sleep apnea patients. Eur Respir J 2017;50:PA4732.

132.Hang LW, Chen CF, Wang CB, et al. The association between continuous positive airway pressure therapy and liver disease development in obstructive sleep apnea/hypopnea syndrome patients: a nationwide population-based cohort study in Taiwan. Sleep Breath 2017;21:461-7.

133. Buttacavoli M, Gruttad'Auria CI, Olivo M, et al. Liver Steatosis and Fibrosis in OSA patients After Long-term CPAP Treatment: A Preliminary Ultrasound Study. Ultrasound Med Biol 2016;42:104-9.

134.Jullian-Desayes I, Tamisier R, Zarski JP, et al. Impact of effective versus sham continuous positive airway pressure on liver injury in obstructive sleep apnoea: Data from randomized trials. Respirology 2016;21:378-85.

135.Labarca G, Cruz R, Jorquera J. Continuous Positive Airway Pressure in Patients With Obstructive Sleep Apnea and Non-Alcoholic Steatohepatitis: A Systematic Review and Meta-Analysis. J Clin Sleep Med 2018;14:133-9.

136. Sookoian S, Pirola CJ. Obstructive sleep apnea is associated with fatty liver and abnormal liver enzymes: a meta-analysis. Obes Surg 2013;23:1815-25.

137. Gagnadoux F, Boursier J, Calès P, et al. Obstructive sleep apnoea and non-alcoholic fatty liver disease: Which patients should be referred to hepatologists? Clin Res Hepatol Gastroenterol 2019;43:e90-e92.

138. Monneret D. Fibromax-based nonalcoholic fatty liver disease in chronic obstructive pulmonary disease patients with obstructive sleep apnea: Methodological considerations. F1000Res 2017;6:1669. 